\title{
ON THE NUMBER OF RATONAL POINTS OF A PLANE ALGEBRAIC CURVE
}

\author{
MASSIMO GIULIETTI
}

\begin{abstract}
The number of $\mathbf{F}_{q}$-rational points of a plane non-singular algebraic curve $\mathcal{X}$ defined over a finite field $\mathbf{F}_{q}$ is computed, provided that the generic point of $\mathcal{X}$ is not an inflexion and that $\mathcal{X}$ is Frobenius non-classical with respect to conics.
\end{abstract}

Keywords: Algebraic curves, Rational points, Frobenius non-classical curves.

\section{InTRODUCTION}

Let $\mathcal{X}$ be a plane non-singular algebraic curve of degree $d$ defined over a finite field $\mathbf{F}_{q}$ of order $q=p^{r}$ with $p$ prime. Let $N$ denote the number of points of $\mathcal{X}$ with coordinates in $\mathbf{F}_{q}$, also called $\mathbf{F}_{q}$-rational points. The Hasse-Weil theorem states that

$$
N=q+1-\sum_{i=1}^{2 g} \alpha_{i}
$$

where $\alpha_{i}$ are certain algebraic integers, and $g=\frac{1}{2} d(d-1)$ is the genus of $\mathcal{X}$. Nevertheless, formulas for $N$ in terms of $d$ and some other projective invariants of $\mathcal{X}$ are only known for few curves, see [5], [12]. For instance, $N=q+1+(d-1)(d-2) \sqrt{q}$ for the Fermat curve $X^{d}+Y^{d}+1=0$ with $\sqrt{q} \equiv-1(\bmod d)$, and $q$ squared, but this formula does not hold true for $q=q_{0}^{m}$ with $m>2$ and $q_{0}^{m-1}+\ldots+q_{0}+1 \equiv-1(\bmod d)$, see [11].

In [6] the authors pointed out that $N=d(q-d+2)$ when $\mathcal{X}$ is Frobenius non-classical, that is the image $\operatorname{Fr}(P)$ of a generic point $P$ of $\mathcal{X}$ under the Frobenius map lies in the tangent line at $P$. Note that for $p>2$ any Frobenius non-classical curve is non-classical in the sense that every point of the curve is an inflexion. An example of a Frobenius non-classical curve is the Fermat curve of degree $d=\sqrt{q}+1$ with $q$ squared, also called Hermitian curve.

In this paper we will be concerned with the case where

A) $\mathcal{X}$ is classical;

2000 Math. Subj. Class.: Primary 14G, Secondary 11G.

This research was performed within the activity of GNSAGA of the Italian CNR, with the financial support of the Italian Ministry MIUR, project "Strutture geometriche, combinatorica e loro applicazioni", PRIN 2001-2002. 
B) $\mathcal{X}$ is Frobenius non-classical with respect to conics, that is $\mathbf{F r}(P)$ lies in the osculating conic $\mathcal{C}_{P}$ to $\mathcal{X}$ at a generic point $P$ of $\mathcal{X}$.

For $p \geq 5$, such a curve $\mathcal{X}$ has the "non-classical" type property that the intersection multiplicity $I\left(\mathcal{X}, \mathcal{C}_{P} ; P\right)$ at a generic point $P$ is a power $p^{\nu}$ of $p$. For $p^{\nu}=\sqrt{q}$, an example of such a curve is the Fermat curve of degree $\frac{1}{2}(\sqrt{q}+1)$ with $q$ squared. Our result is the following theorem.

Theorem 1.1. Let $\mathcal{X}$ be a plane non-singular algebraic curve of degree $d$ defined over a finite field of order $q=p^{r}$ with $p \geq 5$ prime. Assume that $\mathcal{X}$ satisfies conditions $A$ ) and B). If $d<p^{\nu}-1$, then

$$
N=\frac{1}{2}[d(q+5-2 d)-k]
$$

where $k$ denotes the number of non- $\mathbf{F}_{q}$-rational inflexion points $P \in \mathcal{X}$.

\section{Preliminary Results}

An essential tool in the study of the number of $\mathbf{F}_{q}$-rational points of an algebraic curve defined over $\mathbf{F}_{q}$ is the Stöhr-Voloch method. Here, we only summarize the results from [13, Sections 1-2] which play a role in the proof of Theorem 1.1.

Let $\mathcal{X}$ be a plane non-singular algebraic curve of degree $d$ and genus $g$ defined over a finite field $\mathbf{F}_{q}$ of order $q=p^{r}$, with $p$ prime. Let $\overline{\mathbf{F}}_{q}(\mathcal{X})$ be the function field of $\mathcal{X}$, and $x_{0}, x_{1}, x_{2}$ $\mathbf{F}_{q}$-rational functions in $\overline{\mathbf{F}}_{q}(\mathcal{X})$ such that $\mathcal{X}$ has homogeneous equation $F\left(x_{0}, x_{1}, x_{2}\right)=0$. Also, assume that $\mathcal{X}$ is classical, that is the generic point of $\mathcal{X}$ is not an inflexion.

The ramification divisor $R$ and the $\mathbf{F}_{q}$-Frobenius divisor $S$ of $\mathcal{X}$ are defined as follows

$$
\begin{gathered}
R=\operatorname{div}\left(\operatorname{det}\left(\begin{array}{ccc}
x_{0} & x_{1} & x_{2} \\
D_{t}^{1}\left(x_{0}\right) & D_{t}^{1}\left(x_{1}\right) & D_{t}^{1}\left(x_{2}\right) \\
D_{t}^{2}\left(x_{0}\right) & D_{t}^{2}\left(x_{1}\right) & D_{t}^{2}\left(x_{2}\right)
\end{array}\right)\right)+3 \operatorname{div}(d t)+3 E, \\
S=\operatorname{div}\left(\operatorname{det}\left(\begin{array}{ccc}
x_{0}^{q} & x_{1}^{q} & x_{2}^{q} \\
x_{0} & x_{1} & x_{2} \\
D_{t}^{1}\left(x_{0}\right) & D_{t}^{1}\left(x_{1}\right) & D_{t}^{1}\left(x_{2}\right)
\end{array}\right)\right)+\operatorname{div}(d t)+(q+2) E,
\end{gathered}
$$

where $D_{t}^{(k)}$ is the $k$-th Hasse derivative with respect to a separating variable $t$, and $E=$ $\sum v_{P}(E) P$ with $v_{P}(E)=-\min \left\{v_{P}\left(x_{0}\right), v_{P}\left(x_{1}\right), v_{P}\left(x_{2}\right)\right\}$. For $P \in \mathcal{X}$, let $j(P)$ denote the intersection multiplicity of $\mathcal{X}$ with its tangent line at $P$.

Proposition 2.1. (a) $\operatorname{deg} R=3(2 g-2)+3 d$;

(b) $\operatorname{deg} S=(2 g-2)+(q+2) d$;

(c) $v_{P}(R) \geq j(P)-2$; equality holds if and only if $p$ does not divide $j(P)(j(P)-1) / 2$;

(d) $v_{P}(S) \geq j(P)$ for $P \mathbf{F}_{q}$-rational point in $\mathcal{X}$; equality holds if and only if $p$ does not divide $j(P)-1$. 
The order-sequence $\left(j_{0}(P), j_{1}(P), j_{2}(P), j_{3}(P), j_{4}(5), j_{5}(P)\right)$ at $P \in \mathcal{X}$ is defined to be the set of intersection multiplicities of $\mathcal{X}$ at $P$ with conics, arranged in increasing order. Since $\mathcal{X}$ is classical, two cases occur according as $P$ is an inflexion point or not, namely either $(0,1,2,3,4, \epsilon(P))$ or $(0,1,2, j(P), j(P)+1,2 j(P))$. Apart from a finite number of points of $\mathcal{X}$, we have the same order sequence $(0,1,2,3,4, \epsilon)$. If $p \geq 5$ and condition B) is satisfied, then $\epsilon=p^{v}$ for an integer $v \geq 1$, that is $I\left(\mathcal{X}, \mathcal{C}_{P} ; P\right)=\epsilon$ for the osculating conic $\mathcal{C}_{P}$ at $P$. To investigate the number of $\mathbf{F}_{q}$-rational points on $\mathcal{X}$ the following result by Garcia and Voloch [3] is needed.

Proposition 2.2. If $p \geq 5$ and $\mathcal{X}$ if Frobenius non-classical with respect to conics with order sequence $\left(0,1,2,3,4, p^{\nu}\right)$, then there exist $\mathbf{F}_{q}$-rational functions $z_{0}, z_{1}, z_{2}, z_{3}, z_{4}, z_{5} \in$ $\overline{\mathbf{F}}_{q}(\mathcal{X})$ such that

$$
z_{0}^{p^{\nu}} x_{0}^{2}+z_{1}^{p^{\nu}} x_{0} x_{1}+z_{2}^{p^{\nu}} x_{0} x_{2}+z_{3}^{p^{\nu}} x_{1}^{2}+z_{4}^{p^{\nu}} x_{1} x_{2}+z_{5}^{p^{\nu}} x_{2}^{2}=0 .
$$

\section{Curves Which are Frobenius non-Classical For CONiCS}

Throughout this section we assume that $p \geq 5$ and that $\mathcal{X}$ satisfies both conditions $\mathrm{A}$ ) and B). Let $x:=x_{1} / x_{0}, y:=x_{2} / x_{0}$ and $f(x, y)=0$ be a minimal equation of $\mathcal{X}$. By Proposition 2.2, there exist $\mathbf{F}_{q}$-rational functions $z_{0}, z_{1}, z_{2}, z_{3}, z_{4}, z_{5} \in \overline{\mathbf{F}}_{q}(\mathcal{X})$ such that

$$
z_{0}^{p^{\nu}}+z_{1}^{p^{\nu}} x+z_{2}^{p^{\nu}} y+z_{3}^{p^{\nu}} x^{2}+z_{4}^{p^{\nu}} x y+z_{5}^{p^{\nu}} y^{2}=0 .
$$

Let $P=(a, b)$ be an affine point of $\mathcal{X}$, and choose an index $j$ with $0 \leq j \leq 5$ such that $v_{P}\left(z_{j}\right) \leq v_{P}\left(z_{i}\right)$ for $0 \leq i \leq 5$. Putting $m_{i}=z_{i} / z_{j} \in \overline{\mathbf{F}}_{q}(\mathcal{X})$ we have $v_{P}\left(m_{i}\right) \geq 0$, and therefore

$$
m_{0}^{p^{\nu}}+m_{1}^{p^{\nu}} x+m_{2}^{p^{\nu}} y+m_{3}^{p^{\nu}} x^{2}+m_{4}^{p^{\nu}} x y+m_{5}^{p^{\nu}} y^{2}=0,
$$

with $m_{j}=1$. Let $s_{0}=m_{0}(a, b)^{p^{\nu}}+m_{1}(a, b)^{p^{\nu}} x+m_{2}(a, b)^{p^{\nu}} y+m_{3}(a, b)^{p^{\nu}} x^{2}+m_{4}(a, b)^{p^{\nu}} x y+$ $m_{5}(a, b)^{p^{\nu}} y^{2} \in \overline{\mathbf{F}}_{q}(\mathcal{X})$. Then,

$$
\begin{aligned}
s_{0}= & s_{0}-m_{0}^{p^{\nu}}-m_{1}^{p^{\nu}} x-m_{2}^{p^{\nu}} y-m_{3}^{p^{\nu}} x^{2}-m_{4}^{p^{\nu}} x y-m_{5}^{p^{\nu}} y^{2} \\
= & \left(m_{0}(a, b)-m_{0}\right)^{p^{\nu}}+\left(m_{1}(a, b)-m_{1}\right)^{p^{\nu}} x+\left(m_{2}(a, b)-m_{2}\right)^{p^{\nu}} y+\left(m_{3}(a, b)-m_{3}\right)^{p^{\nu}} x^{2} \\
& +\left(m_{4}(a, b)-m_{4}\right)^{p^{\nu}} x y+\left(m_{5}(a, b)-m_{5}\right)^{p^{\nu}} y^{2}
\end{aligned}
$$

and hence $v_{P}\left(s_{0}\right) \geq \min _{0 \leq i \leq 5} v_{P}\left(\left(m_{i}(a, b)-m_{i}\right)^{p^{\nu}}\right) \geq p^{\nu}$. Moreover, as $m_{j}(a, b)=1$, the equation $m_{0}(a, b)^{p^{\nu}}+m_{1}(a, b)^{p^{\nu}} X+m_{2}(a, b)^{p^{\nu}} Y+m_{3}(a, b)^{p^{\nu}} X^{2}+m_{4}(a, b)^{p^{\nu}} X Y+$ $m_{5}(a, b)^{p^{\nu}} Y^{2}=0$ defines a conic. Then the following result is obtained.

Lemma 3.1. For an affine point $P=(a, b)$ of $\mathcal{X}$, let $\mathcal{D}_{P}$ the conic of equation

$m_{0}(a, b)^{p^{\nu}}+m_{1}(a, b)^{p^{\nu}} X+m_{2}(a, b)^{p^{\nu}} Y+m_{3}(a, b)^{p^{\nu}} X^{2}+m_{4}(a, b)^{p^{\nu}} X Y+m_{5}(a, b)^{p^{\nu}} Y^{2}=0$.

Then the intersection multiplicity $I\left(\mathcal{X}, \mathcal{D}_{P} ; P\right)$ is at least $p^{\nu}$.

Proposition 3.2. Let $P=(a, b) \in \mathcal{X}$. If $d<p^{\nu}-1$, then

i) $\mathcal{D}_{P}$ coincides with the osculating conic $\mathcal{C}_{P}$ of $\mathcal{X}$ at $P$; 
ii) $\mathbf{F r}(P) \in \mathcal{C}_{P}$.

Proof. i) If $P$ is not an inflexion point, then the osculating conic $\mathcal{C}_{P}$ is the only conic having intersection multiplicity with $\mathcal{X}$ at $P$ more than 4 , and hence i) holds. If $P$ is an inflexion point, then $j(P) \leq d$. Thus $j_{4}(P) \leq d+1$. Since $d<p^{\nu}-1$, the osculating conic of $\mathcal{X}$ at $P$ turns out to be the only conic having intersection multiplicity with $\mathcal{X}$ at $P$ at least $p^{\nu}$.

ii) First note that since $P$ is an arbitrarily chosen point on $\mathcal{X}$, condition $\mathrm{B}$ ) is not sufficient to prove the assertion. From [13, Corollary 1.3] an equation for $\mathcal{C}_{P}$ is

$$
\operatorname{det}\left(\begin{array}{cccccc}
1 & x & y & x^{2} & x y & y^{2} \\
1 & x(P) & y(P) & x^{2}(P) & x y(P) & y^{2}(P) \\
D_{t}^{j_{1}} 1 & D_{t}^{j_{1}} x(P) & D_{t}^{j_{1}} y(P) & D_{t}^{j_{1}} x^{2}(P) & D_{t}^{j_{1}} x y(P) & D_{t}^{j_{1}} y^{2}(P) \\
D_{t}^{j_{2}} 1 & D_{t}^{j_{2}} x(P) & D_{t}^{j_{2}} y(P) & D_{t}^{j_{2}} x^{2}(P) & D_{t}^{j_{2}} x y(P) & D_{t}^{j_{2}} y^{2}(P) \\
D_{t}^{j_{3}} 1 & D_{t}^{j_{3}} x(P) & D_{t}^{j_{3}} y(P) & D_{t}^{j_{3}} x^{2}(P) & D_{t}^{j_{3}} x y(P) & D_{t}^{j_{3}} y^{2}(P) \\
D_{t}^{j_{4}} 1 & D_{t}^{j_{4}} x(P) & D_{t}^{j_{4}} y(P) & D_{t}^{j_{4}} x^{2}(P) & D_{t}^{j_{4}} x y(P) & D_{t}^{j_{4}} y^{2}(P)
\end{array}\right)=0,
$$

with $t$ local parameter at $P$ and $\left(j_{0}, j_{1}, j_{2}, j_{3}, j_{4}\right)=\left(j_{0}(P), j_{1}(P), j_{2}(P), j_{3}(P), j_{4}(P)\right)$. Then, since $j_{4} \leq \max \{4, j(P)+1\} \leq d+1<p^{\nu}$, from the minimality of the $\mathbf{F}_{q}$-Frobenius orders $([13$, p. 9]) the rational function

$$
\operatorname{det}\left(\begin{array}{cccccc}
1 & x^{q} & y^{q} & \left(x^{q}\right)^{2} & x^{q} y^{q} & \left(y^{q}\right)^{2} \\
1 & x & y & x^{2} & x y & y^{2} \\
D_{t}^{j_{1}} 1 & D_{t}^{j_{1}} x & D_{t}^{j_{1}} y & D_{t}^{j_{1}} x^{2} & D_{t}^{j_{1}} x y & D_{t}^{j_{1}} y^{2} \\
D_{t}^{j_{2}} 1 & D_{t}^{j_{2}} x & D_{t}^{j_{2}} y & D_{t}^{j_{2}} x^{2} & D_{t}^{j_{2}} x y & D_{t}^{j_{2}} y^{2} \\
D_{t}^{j_{3}} 1 & D_{t}^{j_{3}} x & D_{t}^{j_{3}} y & D_{t}^{j_{3}} x^{2} & D_{t}^{j_{3}} x y & D_{t}^{j_{3}} y^{2} \\
D_{t}^{j_{4}} 1 & D_{t}^{j_{4}} x & D_{t}^{j_{4}} y & D_{t}^{j_{4}} x^{2} & D_{t}^{j_{4}} x y & D_{t}^{j_{4}} y^{2}
\end{array}\right)
$$

is equal to 0 . Therefore $\left(a^{q}, b^{q}\right)$ satisfies equation (3.2) and the assertion follows.

The following proposition is the key fact to prove Theorem 1.1.

Proposition 3.3. If $d<p^{\nu}-1$, then $j(P) \in\left\{2,\left(p^{\nu}+1\right) / 2\right\}$ for any point $P \in \mathcal{X}$.

Proof. Let $P=(a, b)$ be a point on $\mathcal{X}$. Introduce a new affine frame $\left(X^{\prime}, Y^{\prime}\right)$ such that $P$ is taken to the origin and the tangent line of $\mathcal{X}$ at $P$ to the $X^{\prime}$-axis. The corresponding change of coordinate functions from $(x, y)$ to $(\xi, \eta)$ is given by

$$
\begin{aligned}
& x=m_{11} \xi+m_{12} \eta+a, \\
& y=m_{21} \xi+m_{22} \eta+b,
\end{aligned}
$$

for some $m_{i j} \in \overline{\mathbf{F}}_{q}, i, j=1,2$. Equation (3.1) is invariant under this transformation. To see this, put, for $0 \leq i \leq 5$,

$$
\begin{aligned}
& z_{i}(x, y)=z_{i}\left(m_{11} \xi+m_{12} \eta+a, m_{21} \xi+m_{22} \eta+b\right)=\bar{z}_{i}(\xi, \eta), \\
& a=c^{p^{\nu}}, \quad b=d^{p^{\nu}}, \quad m_{i j}=n_{i j}^{p^{\nu}}, i, j=1,2,
\end{aligned}
$$


and write $\bar{z}_{i}=\bar{z}_{i}(\xi, \eta)$. Then, with

$$
\begin{aligned}
& \zeta_{0}(\xi, \eta)=\bar{z}_{0}+c \bar{z}_{1}+d \bar{z}_{2}+c^{2} \bar{z}_{3}+c d \bar{z}_{4}+d^{2} \bar{z}_{5}, \\
& \zeta_{1}(\xi, \eta)=n_{11} \bar{z}_{1}+n_{21} \bar{z}_{2}+2 c n_{11} \bar{z}_{3}+\left(c n_{11}+d n_{21}\right) \bar{z}_{4}+2 d n_{21} \bar{z}_{5}, \\
& \zeta_{2}(\xi, \eta)=n_{12} \bar{z}_{1}+n_{22} \bar{z}_{2}+2 c n_{12} \bar{z}_{3}+\left(c n_{22}+d n_{12}\right) \bar{z}_{4}+2 d n_{22} \bar{z}_{5}, \\
& \zeta_{3}(\xi, \eta)=n_{11}^{2} \bar{z}_{3}+n_{11} n_{21} \bar{z}_{4}+n_{21}^{2} \bar{z}_{5}, \\
& \zeta_{4}(\xi, \eta)=2 n_{11} n_{12} \bar{z}_{3}+\left(n_{12} n_{21}+n_{11} n_{22}\right) \bar{z}_{4}+2 n_{21} n_{22} \bar{z}_{5}, \\
& \zeta_{5}(\xi, \eta)=n_{12}^{2} \bar{z}_{3}+n_{12} n_{22} \bar{z}_{4}+n_{22}^{2} \bar{z}_{5},
\end{aligned}
$$

equation (3.1) becomes

$$
\zeta_{0}^{p^{\nu}}+\zeta_{1}^{p^{\nu}} \xi+\zeta_{2}^{p^{\nu}} \eta+_{3}^{p^{\nu}} \xi^{2}+\zeta_{4}^{p^{\nu}} \xi \eta+\zeta_{5}^{p^{\nu}} \eta^{2}=0
$$

Since the tangent line to $\mathcal{X}$ at $P^{\prime}=(0,0)$ has equation $Y^{\prime}=0$, we have $v_{P^{\prime}}(\eta)=j(P)$. Let $v_{P^{\prime}}\left(\zeta_{i}\right)=k_{i}, i=0,1, \ldots, 5$. The left-hand side in (3.4) is the sum of six rational functions with valuations at $P^{\prime}$ :

$$
\begin{array}{cc}
v_{P^{\prime}}\left(\zeta_{0}^{p^{\nu}}\right)=k_{0} p^{\nu}, & v_{P^{\prime}}\left(\zeta_{1}^{p^{\nu}} \xi\right)=k_{1} p^{\nu}+1, \\
v_{P^{\prime}}\left(\zeta_{2}^{p^{\nu}} \eta\right)=k_{2} p^{\nu}+j(P), & v_{P^{\prime}}\left(\zeta_{3}^{p^{\nu}} \xi^{2}\right)=k_{3} p^{\nu}+2, \\
v_{P^{\prime}}\left(\zeta_{4}^{p^{\nu}} \xi \eta\right)=k_{4} p^{\nu}+1+j(P), & v_{P^{\prime}}\left(\zeta_{5}^{p^{\nu}} \eta^{2}\right)=k_{5} p^{\nu}+2 j(P) .
\end{array}
$$

At least two of these values must be equal, and less than or equal to the remaining four. Hence one of the following holds:

$$
\begin{aligned}
& \left(k_{0}-k_{1}\right) p^{\nu}=1, \quad\left(k_{1}-k_{3}\right) p^{\nu}=1, \quad\left(k_{2}-k_{4}\right) p^{\nu}=1, \\
& \left(k_{0}-k_{3}\right) p^{\nu}=2, \quad\left(k_{0}-k_{2}\right) p^{\nu}=j(P), \quad\left(k_{2}-k_{5}\right) p^{\nu}=j(P), \\
& \left(k_{0}-k_{4}\right) p^{\nu}=1+j(P), \quad\left(k_{0}-k_{5}\right) p^{\nu}=2 j(P), \quad\left(k_{1}-k_{2}\right) p^{\nu}=j(P)-1, \\
& \left(k_{3}-k_{4}\right) p^{\nu}=j(P)-1, \quad\left(k_{1}-k_{4}\right) p^{\nu}=j(P), \quad\left(k_{4}-k_{5}\right) p^{\nu}=j(P)-1, \\
& \left(k_{1}-k_{5}\right) p^{\nu}=2 j(P)-1, \quad\left(k_{2}-k_{3}\right) p^{\nu}=2-j(P), \quad\left(k_{3}-k_{5}\right) p^{\nu}=2 j(P)-2,
\end{aligned}
$$

Since $d<p^{\nu}-1$ we have $1+j(P)<p^{\nu}$. This leaves just two possibilities: $j(P)=2$, $j(P)=\left(p^{\nu}+1\right) / 2$.

\section{Proof of Theorem 1.1}

To estimate the number of $\mathbf{F}_{q}$-rational points of $\mathcal{X}$, we will use a procedure similar to that in [6]. To do this, we go on to study the ramification divisor $R$ and the $\mathbf{F}_{q}$-Frobenius divisor $S$ of $\mathcal{X}$.

Lemma 4.1. If $d<p^{\nu}-1$, then for a point $P \in \mathcal{X}$

$$
v_{P}(R)=j(P)-2, \quad v_{P}(S)= \begin{cases}j(P) & \text { if } P \in \mathcal{X}\left(\mathbf{F}_{q}\right), \\ 0 & \text { if } P \notin \mathcal{X}\left(\mathbf{F}_{q}\right) \text { and } j(P)=2, \\ j(P)-1 & \text { if } P \notin \mathcal{X}\left(\mathbf{F}_{q}\right) \text { and } j(P)>2 .\end{cases}
$$


Proof. From Proposition $3.3 j(P)=2$ or $j(P)=(1 / 2)\left(p^{\nu}+1\right)$, hence $v_{P}(R)=j(P)-2$ by (c) of Proposition 2.1. Suppose now that $P \in \mathcal{X}\left(\mathbf{F}_{q}\right)$. Since $p$ does not divide $(j(P)-1)$, from (d) of Proposition 2.1 it follows $v_{P}(S)=j(P)$. For $P \notin \mathcal{X}\left(\mathbf{F}_{q}\right)$, we distinguish two cases.

If $j(P)=2$, any degenerate conic meet $\mathcal{X}$ at $P=(a, b)$ with multiplicity at most 4 , and therefore the osculating conic $\mathcal{C}_{P}$ at $P$ is irreducible. Moreover, $\left(a^{q}, b^{q}\right)$ belongs to $\mathcal{C}_{P}$ by ii) of Propoposition 3.2. Then $v_{P}(S)=0$ since otherwise $\left(a^{q}, b^{q}\right)$ would belong to the tangent line $l_{P}$ at $P$, and there would exist too many intersections between $l_{P}$ and $\mathcal{C}_{P}$.

Suppose now that $j(P)>2$. Note that the osculating conic $\mathcal{C}_{P}$ is the tangent line $l_{P}$ counted twice. From Proposition 3.2 it follows that $\left(a^{q}, b^{q}\right) \in \mathcal{C}_{P}$ and hence $\left(a^{q}, b^{q}\right) \in l_{P}$. Now, from equation (2.1), $v_{P}(S)=v_{P}\left(\left(x-x^{q}\right) D_{t}^{1} y-\left(y-y^{q}\right) D_{t}^{1} x\right)=v_{P}\left(\left(x-x^{q}\right) d y / d t-\right.$ $\left.\left(y-y^{q}\right) d x / d t\right)$, with a separating variable $t \in \mathbf{F}_{q}(x, y)$ such that $v_{P}(d t)=0$. Since $v_{P}(S)$ is not invariant under all affine transformations but only for those fixing the plane over $\mathbf{F}_{q}$, it is necessary to see how $v_{P}(S)$ changes under an $\overline{\mathbf{F}}_{q}$-linear transformation. With $x, y, \xi, \eta$ as in (3.3),

$$
\begin{aligned}
\left(x-x^{q}\right) d y-\left(y-y^{q}\right) d x= & {\left[\left(a-a^{q}\right) m_{21}-\left(b-b^{q}\right) m_{11}\right] d \xi } \\
& +\left[\left(a-a^{q}\right) m_{22}-\left(b-b^{q}\right) m_{12}\right] d \eta \\
& +\left(m_{11} m_{22}-m_{12} m_{21}\right)(\xi d \eta-\eta d \xi) \\
& -\left(m_{11} \xi+m_{12} \eta\right)^{q}\left(m_{21} d \xi+m_{22} d \eta\right) \\
& +\left(m_{21} \xi+m_{22} \eta\right)^{q}\left(m_{11} d \xi+m_{12} d \eta\right) .
\end{aligned}
$$

Now we let $\tau=t(\xi, \eta) \in \overline{\mathbf{F}}_{q}(\mathcal{X})$. By letting $\xi^{\prime}=d \xi / d \tau$ and $\eta^{\prime}=d \eta / d \tau$, the following formula is arrived at:

$$
\begin{aligned}
v_{P}(S)= & v_{P^{\prime}}\left(\xi^{\prime}\right)+v_{P^{\prime}}\left\{\left[\left(a-a^{q}\right) m_{21}-\left(b-b^{q}\right) m_{11}\right]\right. \\
& +\left[\left(a-a^{q}\right) m_{22}-\left(b-b^{q}\right) m_{12}\right] \eta^{\prime} / \xi^{\prime} \\
& +\left(m_{11} m_{22}-m_{12} m_{21}\right)\left(\xi \eta^{\prime} / \xi^{\prime}-\eta\right) \\
& -\left(m_{11} \xi+m_{12} \eta\right)^{q}\left(m_{21}+m_{22} \eta^{\prime} / \xi^{\prime}\right) \\
& \left.+\left(m_{21} \xi+m_{22} \eta\right)^{q}\left(m_{11}+m_{12} \eta^{\prime} / \xi^{\prime}\right)\right\}
\end{aligned}
$$

Note that $v_{P^{\prime}}(\xi)=1$ and $v_{P^{\prime}}(\eta)=j(P)$ are both prime to $p$ by Proposition 3.3. Hence, $v_{P^{\prime}}\left(\xi^{\prime}\right)=0$ and $v_{P^{\prime}}\left(\eta^{\prime}\right)=j(P)-1$. Furthermore, $\left(a-a^{q}\right) m_{21}-\left(b-b^{q}\right) m_{11}=0$ and $\left(a-a^{q}\right) m_{22}-\left(b-b^{q}\right) m_{12} \neq 0$, as the line joining $(a, b)$ and $\left(a^{q}, b^{q}\right)$ is the tangent line at $P$. Hence $v_{P}(S)=v_{P^{\prime}}\left(\eta^{\prime} / \xi^{\prime}\right)=j(P)-1$.

Now we can prove the main result of the paper.

\section{Proof of Theorem 1.1}

Proof. The genus $g$ of $\mathcal{X}$ is equal to $\frac{(d-1)(d-2)}{2}$, hence $\operatorname{deg}(R)=3 d(d-3)+3 d$ and $\operatorname{deg}(S)=$ $d(d-3)+d(q+2)$. Therefore $d(q+5-2 d)=\operatorname{deg}(S)-\operatorname{deg}(R)=\sum_{P \in \mathcal{X}}\left[v_{P}(S)-v_{P}(R)\right]$. Then the assertion follows from Lemma 4.1. 
Remark 4.2. Note that the proofs of Lemma 4.1 and of Theorem 1.1 depend on conditions A) and B), and on the following two facts arising from $d<p^{\nu}-1$ :

1) $\mathbf{F} r(P) \in \mathcal{C}_{P}$ for every $P \in \mathcal{X}$ (see Proposition 3.2);

2) $p$ does not divide $j(P)(j(P)-1)$ for every $P \in \mathcal{X}$ (see Proposition 3.3).

Therefore, if $\mathcal{X}$ fulfills the above two conditions together with A) and B) then its number of $\mathbf{F}_{q}$-rational points is given by equation (1.1). This happens for instance for the following Fermat curves $\alpha X^{d}+\beta Y^{d}=1$ defined over $\mathbf{F}_{q}$ (see [4, Thm. 2, Thm. 3]):

i) $d=(q-1) / 2\left(p^{r}-1\right)$ with $\alpha^{2}, \beta^{2} \in \mathbf{F}_{p^{r}} \backslash\{0\}$;

ii) $d=2(q-1) /\left(p^{r}-1\right)$ with $p \equiv 1(\bmod 4)$ and $\alpha, \beta$ non-zero squares in $\mathbf{F}_{p^{r}}$.

Remark 4.3. An example of plane non-singular algebraic curve which satisfies conditions A) and B) but not equation (1.1) is given by the Fermat curve $\alpha X^{d}+\beta Y^{d}=1$, with $d=2(q-1) /\left(p^{r}-1\right)$ and $\alpha, \beta$ non-squares in $\mathbf{F}_{p^{r}}$. The number $N$ of its $\mathbf{F}_{q^{-} \text {-rational points }}$

is $\frac{1}{2} d(q-1+d-d \psi+2 \psi)$, where $\psi=0$ for $r$ odd and $p \equiv 3(\bmod 4), \psi=1$ otherwise (see [4. Example (viii)]). Equation (1.1) would instead give $N=\frac{1}{2} d(q+2-2 d+\psi)$. It is easily seen that such a curve does not satisfy condition 1) in Remark 4.2.

\section{ACKNOWLEDGMENTS}

The author would like to thank Prof. G. Korchmáros and Prof. F. Torres for their useful comments.

\section{REFERENCES}

[1] A. Aguglia and G. Korchmáros, On the number of rational points of an algebraic curve over finite fields, Bull. Belg. Math. Soc 7 (2000), 333-342.

[2] A. Garcia and P. Viana, Weierstrass points on certain non-classical curves, Arch. Math. 46 (1986), $315-322$.

[3] A. Garcia and J.F. Voloch, Wronskians and linear independence in fields of prime characteristic, Manuscripta Math. 59 (1987), 457-469.

[4] A. Garcia and J.F. Voloch, Fermat curves over finite fields, J. Number Theory 30 (1988), 345-356.

[5] G. van der Geer and M. van der Vlugt, Tables of curves with many points, January 2002, http://www.wins.uva.nl/ geer.

[6] A. Hefez and J.F. Voloch, Frobenius non classical curves, Arch. Math. 54 (1990), 263-273.

[7] J.W.P. Hirschfeld, Projective Geometries Over Finite Fields, second edition, Oxford University Press, Oxford, 1998.

[8] J.W.P. Hirschfeld and G. Korchmáros, Embedding an arc into a conic in a finite plane, Finite Fields Appl. 2 (1996), 274-292.

[9] J.W.P. Hirschfeld and G. Korchmáros, On the number of rational points on an algebraic curve over a finite field, Bull. Belg. Math. Soc. 5(2-3) (1998), 313-340.

[10] J.W.P. Hirschfeld and G. Korchmáros, Arcs and curves over a finite field, Finite Fields Appl. 5 (1999), 393-408.

[11] G. Korchmáros and T. Szőnyi, Fermat Curves over Finite Fields and Cyclic Subests in HighDimensional Projective Spaces, Finite Fields Appl. 5 (1999), 206-217. 
[12] K. Lauter, Geometric methods for improving the upper bounds on the number of rational points on algebraic curves over finite fields with an appendix by Jean-Pierre Serre, J. Alg. Geometry 10 (2001), 19-36.

[13] K.O. Stöhr and J.F. Voloch, Weierstrass points and curves over finite fields, Proc. London Math. Soc. 52 (1986), 1-19.

Dipartimento di Matematica e Informatica, Università degli Studi di Perugia, Via VanVitelli, 1, 06123 Perugia, Italy 